\title{
PGD for hereditary breast and ovarian cancer: the route to universal tests for $B R C A 1$ and BRCA2 mutation carriers
}

\author{
Marion Drüsedau ${ }^{1,6}$, Jos C Dreesen ${ }^{1,2,6}$, Inge Derks-Smeets ${ }^{1,2}$, Edith Coonen ${ }^{3}$, Ron van Golde ${ }^{2,3}$, \\ Jannie van Echten-Arends ${ }^{4}$, Peter MM Kastrop ${ }^{5}$, Marinus J Blok ${ }^{1,2}$, Encarna Gómez-García ${ }^{1,2}$, \\ Joep P Geraedts ${ }^{1,2}$, Hubert J Smeets ${ }^{1,2}$, Christine E de Die-Smulders ${ }^{1,2}$ and Aimée D Paulussen ${ }^{\star 1,2}$
}

Preimplantation Genetic Diagnosis (PGD) is a method of testing in vitro embryos as an alternative to prenatal diagnosis with possible termination of pregnancy in case of an affected child. Recently, PGD for hereditary breast and ovarian cancer caused by BRCA1 and BRCA2 mutations has found its way in specialized labs. We describe the route to universal single-cell PGD tests for carriers of $B R C A 1 / 2$ mutations. Originally, mutation-specific protocols with one or two markers were set up and changed when new couples were not informative. This route of changing protocols was finalized after 2 years with universal tests for both BRCA1 and BRCA2 mutation carriers based on haplotyping of, respectively, 6 (BRCA1) and 8 (BRCA2) microsatellite markers in a multiplex PCR. Using all protocols, 30 couples had a total of 47 PGD cycles performed. Eight cycles were cancelled upon IVF treatment due to hypostimulation. Of the remaining 39 cycles, a total of 261 embryos were biopsied and a genetic diagnosis was obtained in 244 (93\%). In 34 of the 39 cycles (84.6\%), an embryo transfer was possible and resulted in 8 pregnancies leading to a fetal heart beat per oocyte retrieval of $20.5 \%$ and a fetal heart beat per embryonic transfer of $\mathbf{2 3 . 5} \%$. The preparation time and costs for set-up and validation of tests are minimized. The informativity of microsatellite markers used in the universal PGD-PCR tests is based on CEPH and deCODE pedigrees, making the tests applicable in 90\% of couples coming from these populations.

European Journal of Human Genetics (2013) 21, 1361-1368; doi:10.1038/ejhg.2013.50; published online 27 March 2013

Keywords: PGD; HBOC; BRCA1; BRCA2; microsatellites; mutation

\section{INTRODUCTION}

In the Netherlands, the lifetime risk for women to develop breast cancer is one in eight, leading to a diagnosis of breast cancer in about 12000 women each year. Breast cancer is thereby the most prevalent form of cancer in women in the Netherlands. In about $5-10 \%$ of the total hereditary breast and ovarian cancer (HBOC) patients, the mode of inheritance is autosomal dominant with incomplete penetrance. ${ }^{1}$ The majority of this heritability is explained by mutations in genes BRCA1 (MIM:113705, Genbank:U14680) and BRCA2 (MIM:600185, Genbank:U43746), located on chromosomes 17q21 and 13q12.3, respectively. Both BRCA proteins act as tumor-suppressor genes and have, in this role, been shown to function in transcriptional regulation, DNA repair, DNA recombination and cell-cycle checkpoint control, thereby explaining how heterozygous loss of these genes can contribute to cancer initiation and progression. ${ }^{2}$ Since the discovery of the two BRCA genes in $1994^{3}$ and $1995,{ }^{4}>1500$ different mutations for the BRCA1 gene and > 1200 mutations for the BRCA2 gene have been identified in patients worldwide (Human Gene Mutation Database: www.hgmd.cf.ac.uk). Several founder mutations have been identified in specific ethnic populations as the Ashkenazi Jews ${ }^{5}$ or in specific regions/countries, such as Norway, ${ }^{6}$ Poland ${ }^{7}$ and China. ${ }^{8}$ Even though in the Netherlands there have been some regional founder mutations, ${ }^{9}$ the majority of mutations detected in BRCA1 and BRCA2 genes represent private mutations.

Preimplantation Genetic Diagnosis (PGD) was introduced into the clinic at the Maastricht University Medical Center $\left(\mathrm{MUMC}^{+}\right)$in 1995. ${ }^{10}$ The introduction of PGD for HBOC resulted in lots of debate and discussion due to the late onset, incomplete penetrance and availability of preventive and therapeutic options. PGD for HBOC is now permitted in some countries as the UK, ${ }^{11}$ Israel, $^{12}$ Belgium ${ }^{13}$ and the Netherlands. Due to the variety of mutations carried by the different PGD applicants, mainly mutation specific tests or tests with few markers are described. As we experienced the continuing need for protocol adjustments, we aimed at designing universal PGD protocols for BRCA1/2 mutation carriers. The methodology applied in these universal protocols is based on genetic linkage, using highly informative microsatellite markers in the close vicinity of the BRCA1 and $B R C A 2$ genes that are likely inherited together during meiosis.

${ }^{1}$ Department of Clinical Genetics, University Medical Centre, Maastricht, The Netherlands; ${ }^{2}$ School for Oncology and Developmental Biology (GROW), University Medical Centre, Maastricht, The Netherlands; ${ }^{3}$ Department of Obstetrics and Gynaecology (IVF Laboratory), University Medical Centre, Maastricht, The Netherlands; ${ }^{4}$ Department of Obstetrics and Gynaecology, University of Groningen, University Medical Center Groningen, Groningen, The Netherlands; ${ }^{5}$ Department of Reproductive Medicine, University Medical Center Utrecht, Utrecht, The Netherlands

Joint first author.

*Correspondence: Dr AD Paulussen, Department of Clinical Genetics, Maastricht University Medical Centre, Postbus 5800, Maastricht 6202 AZ, The Netherlands. Tel: +31 43 3875843; Fax: +31 43 3877901; E-mail: aimee.paulussen@mumc.nl

Received 11 October 2012; revised 21 February 2013; accepted 22 February 2013; published online 27 March 2013 
Using this methodology, the need to incorporate the specific familial mutation is omitted; however, at least two informative family members (meiosis) are needed to definitely determine the 'risk' haplotype. These universal protocols reduce the patients' waiting time as well as set-up and validation costs.

\section{PATIENTS AND METHODS}

\section{PGD couples and counseling}

Thirty couples applied for a PGD procedure for HBOC between 2009 and 2011. Verbal and written information regarding the procedure, including in vitro fertilization (IVF) and intracytoplasmic sperm injection (ICSI), the risks and complications of IVF, PGD, the risk of misdiagnosis in PGD, the success rate of the treatment and the health of children born after PGD, was provided by a clinical geneticist or PGD physician and/or a gynecologist. The safety of ovarian stimulation for IVF treatment in BRCA-positive women was discussed and counselors explained that current knowledge does not suggest a significant increased risk for breast cancer in these women. ${ }^{14}$ Fifteen couples were BRCA1 and 15 couples were BRCA2. In 60\% (9/15) of both $B R C A 1$ and BRCA2 couples, the female carrier was the index case (Table 1). The average age of the women at the start of the first cycle was 30.5 years for BRCA1 and 30.7 years for BRCA2. Three of the 17 women had had breast cancer before starting with the PGD procedure; all others had undergone pre-symptomatic testing because of an affected relative.

\section{Design of the PGD protocols}

Microsatellite markers in or in the near vicinity of the BRCA1 and BRCA2 gene loci were explored using free accessible databases (c.q. NCBI; http:// www.ncbi.nlm.nih.gov, UCSC; http://genome.ucsc.edu). This exploration of markers provided a list of approximately 25-30 microsatellite markers flanking the BRCA1 and BRCA2 loci (10-15 on either side of the locus). An overview of the developed protocols is summarized in Figure 1. Positions of markers relative to the BRCA1/2 loci are depicted in Figure 2. During the process of protocol set-up and validation, the final selection of markers for the universal protocols were made based on heterozygosity/informativity of the markers, redundancy with others markers, competition with other markers in the multiplex PCR and percentages of allelic drop-out (ADO). For the universal $B R C A 1$ protocol, six informative markers were selected: three proximal, one intragenic and two distal to the BRCA1 locus (Figure 2, universal markers underlined). The genetic distance between the outer markers is $2.1 \mathrm{~cm}$, according to Genethon and $2.14 \mathrm{~cm}$ accrding to Marshfield genetic maps (deCODE not available). The average heterozygosity of markers in the universal protocol is 0.77 . For the $B R C A 2$ protocol, eight informative markers were selected: four proximal and four distal to the BRCA2 locus (Figure 2). The genetic distance between the outer markers is $4.65 \mathrm{~cm}$ according to deCODE, $5.4 \mathrm{~cm}$ according to Genethon and $3.36 \mathrm{~cm}$ according to Marshfield genetic maps. The average heterozygosity of the markers in the universal protocol is 0.74 . For a detailed description of the primers used, the fluorescent labels and PCR product lengths, see Table 2. For non-informative PGD couples or couples for whom the risk haplotype could not be established by at least two meioses, a mutation-specific protocol was designed by detection of the private mutation combined with at least one informative marker. In these tests, single substitution mutations were detected using the difference in fragment length in case of base pair deletions/insertions or using the double allele amplification refractory mutation systems technique. ${ }^{15}$

\section{Validation of PGD protocols}

The primers for the described microsatellite markers were developed using the free web program 'primer3 Input' (http://frodo.wi.mit.edu/primer3/). Criteria for primer design were primer size (20-30 nucleotides), GC content (40-60\%) and $\mathrm{T}_{\mathrm{m}}$ of the primers $\left(60-70^{\circ} \mathrm{C}\right.$, with maximum difference of $\left.4{ }^{\circ} \mathrm{C}\right)$, primers preferably ending $3^{\prime}$ with a guanine or cytosine and PCR product lengths $<300 \mathrm{bp}$. Fluorescent labels were designed in such a way that PCR products with the same label did not overlap. All primers were mixed in one multiplex PCR following the ESHRE guidelines. ${ }^{16}$ After optimization of the single-cell multiplex PCR, all protocols were validated by testing at least 50 single leucocytes heterozygous for each marker in at least three separate experiments to assess amplification efficiency and ADO rate. Additionally 15-20 PCR blanks were analyzed to determine contamination. Only amplification efficiency rates $>90 \%$ and $\mathrm{ADO}$ rates $<10 \%$ were acceptable for implementation in the single-cell protocol.

\section{IVF/ICSI/PGD procedure}

Controlled ovarian hyperstimulation was performed as described earlier. ${ }^{17}$ Oocytes were retrieved under ultrasound guidance. After $5 \mathrm{~h}$ of maturation, MII oocytes were fertilized by means of ICSI ${ }^{18}$ followed by embryo culturing. ${ }^{19}$ Embryo morphology grade was used as one of the parameters to assess the embryo quality. On the morning of day 3 post fertilization, blastomeres were biopsied from cleavage stage embryos. From each cleavage-stage embryo, one ( $4-7$ cells) or two blastomeres ( $>8$ cell stage) were biopsied according to the ESHRE PGD guidelines. ${ }^{20}$ Of 4-7 cell cleavage-stage embryos, only once cell was biopsied as this has been a general policy in our IVF centre for $>15$ years. Rationale for biopsying two cells is to increase the number of conclusive genetic results, in case of (partly) PCR failure. Biopsied blastomeres were rinsed three times in washing buffer $\left(\mathrm{Ca}^{2+}\right.$ - and $\mathrm{Mg}^{2+}$-free phosphatebuffered saline with $1 \%$ polyvinylpyrrolidone (Sigma-Aldrich Chemie BV, Zwijndrecht, The Netherlands) and $0.1 \mathrm{mg} / \mathrm{ml}$ Phenol Red (Sigma-Aldrich Chemie BV)) and $2 \mu \mathrm{l}$ washing buffer was transferred into 0.2-ml PCR tubes. Blank samples, containing $2 \mu \mathrm{l}$ of the last washing droplet, were collected for each blastomere to monitor contamination. Single blastomere and blank samples were stored at least for $20 \mathrm{~min}$ at $-20^{\circ} \mathrm{C}$, until the PCR was performed.

\section{PCR and genescan analysis}

Single cells (blastomeres and leukocytes) were lysed by incubation at $65^{\circ} \mathrm{C}$ for $10 \mathrm{~min}$. in lysisbuffer (50 mM DTT and $200 \mathrm{~mm} \mathrm{NaOH}$ ) before amplification. Multiplex PCR for the polymorphic markers and allele-specific PCR for a private mutation contained $1 \times$ QIAGEN Multiplex PCR Master Mix (QIAGEN, Venlo, the Netherlands), $20 \mathrm{mmol} / \mathrm{l}$ Tricine (Sigma) and primers (Applied Biosystems, Warrington, UK) in a final volume of $25 \mu \mathrm{l}$. The primer concentrations of the individual sets in the multiplexed PCRs varied between $100 \mathrm{~nm}$ and $1 \mathrm{~mm}$ per primer pair. All PCR reactions were performed with an initial activation step of $15 \mathrm{~min}$ denaturation at $95^{\circ} \mathrm{C}$. The denaturationannealing-elongation cycles for the BRCA1 multiplex PCR were 42 cycles of $10 \mathrm{~s}$ at $95^{\circ} \mathrm{C}-90 \mathrm{~s}$ at $62^{\circ} \mathrm{C}-60 \mathrm{~s}$ at $72^{\circ} \mathrm{C}$ and for the BRCA2 multiplex 10 initial cycles of $10 \mathrm{~s}$ at $95^{\circ} \mathrm{C}-60 \mathrm{~s}$ at $63^{\circ} \mathrm{C}-60 \mathrm{~s}$ at $72^{\circ} \mathrm{C}$ followed by 32 cycles of $10 \mathrm{~s}$ at $95^{\circ} \mathrm{C}-45 \mathrm{~s}$ at $63^{\circ} \mathrm{C}-60 \mathrm{~s}$ at $72^{\circ} \mathrm{C}$. The PCR products were diluted $(10 \times B R C A 1$ PCR, $20 \times B R C A 2$ PCR) and separated based on fragment length using capillary electrophoresis on an ABI PRISM 3730 Genetic Analyzer (Applied Biosystems). Fragment lengths were analyzed using the GeneMapper software provided by the manufacturer. For an example of genescan analysis and the corresponding haplotypes, see Figure 3.

\section{Quality assessment}

After genetic diagnosis of embryos and embryo transfer of one or two healthy embryo(s) into the uterus and cryopreservation of the remaining unaffected, good quality, embryos, the remaining embryos were collected for quality assessment purposes after informed consent of the couple involved. Usually, the total embryo is selected as a whole to confirm the genetic diagnosis made during the PGD cycle. In a minority of cases, embryos were split into single blastomeres and analyzed separately. Of the 30 PGD couples, the untransferred embryos of 16 couples ( 8 BRCA1, 8 BRCA2) were collected to determine accuracy of genetic diagnosis/misdiagnosis rate.

\section{RESULTS PGD protocols}

Five protocols for $B R C A 1$ and four protocols for $B R C A 2$ have been developed and validated at the single-cell level (Figures 1a and b). For $B R C A 1$, we started with the first protocol of three markers of which two were intragenic and one distal (protocol 1, Figure 1a). The next couple had too few family members and the risk haplotype could not 


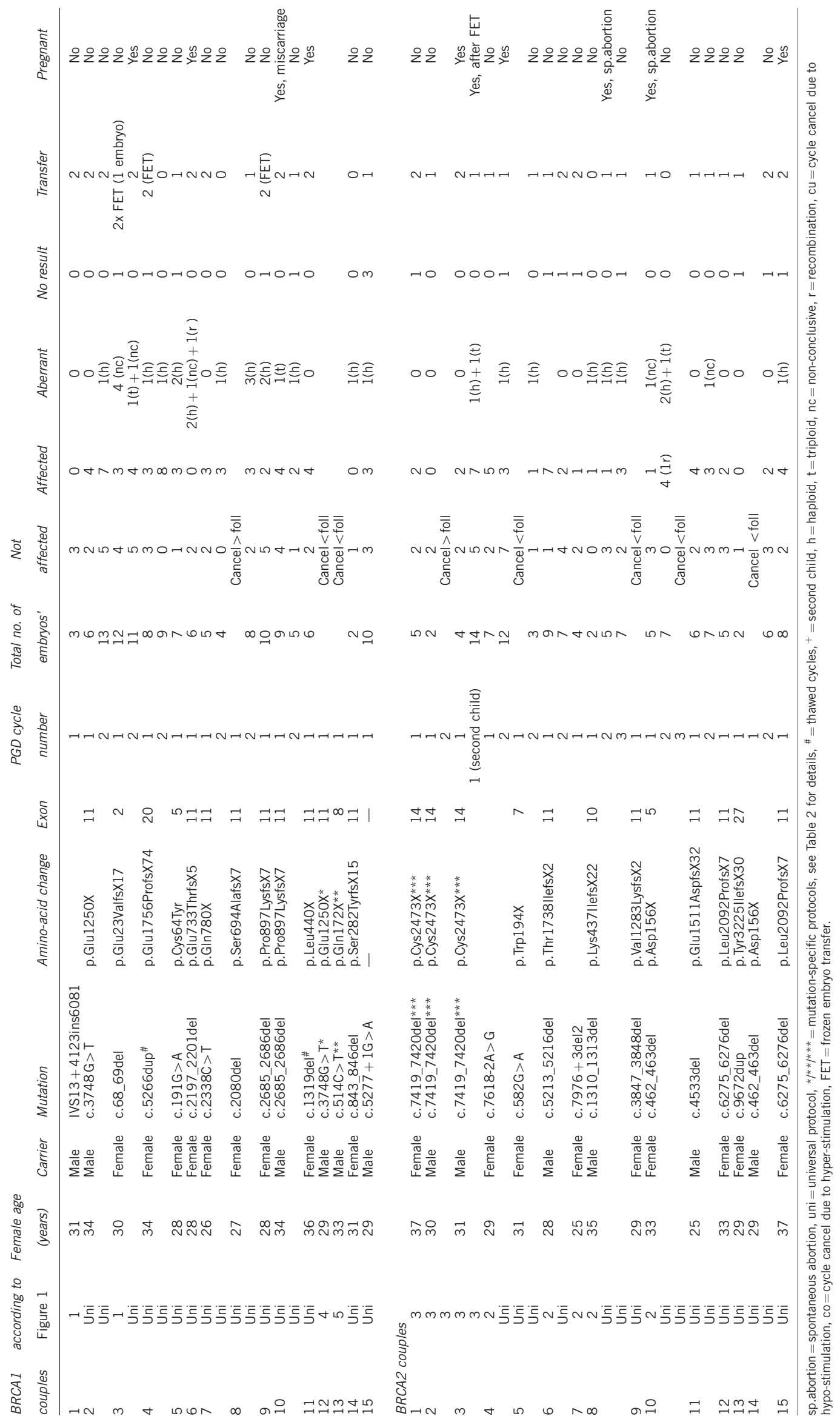



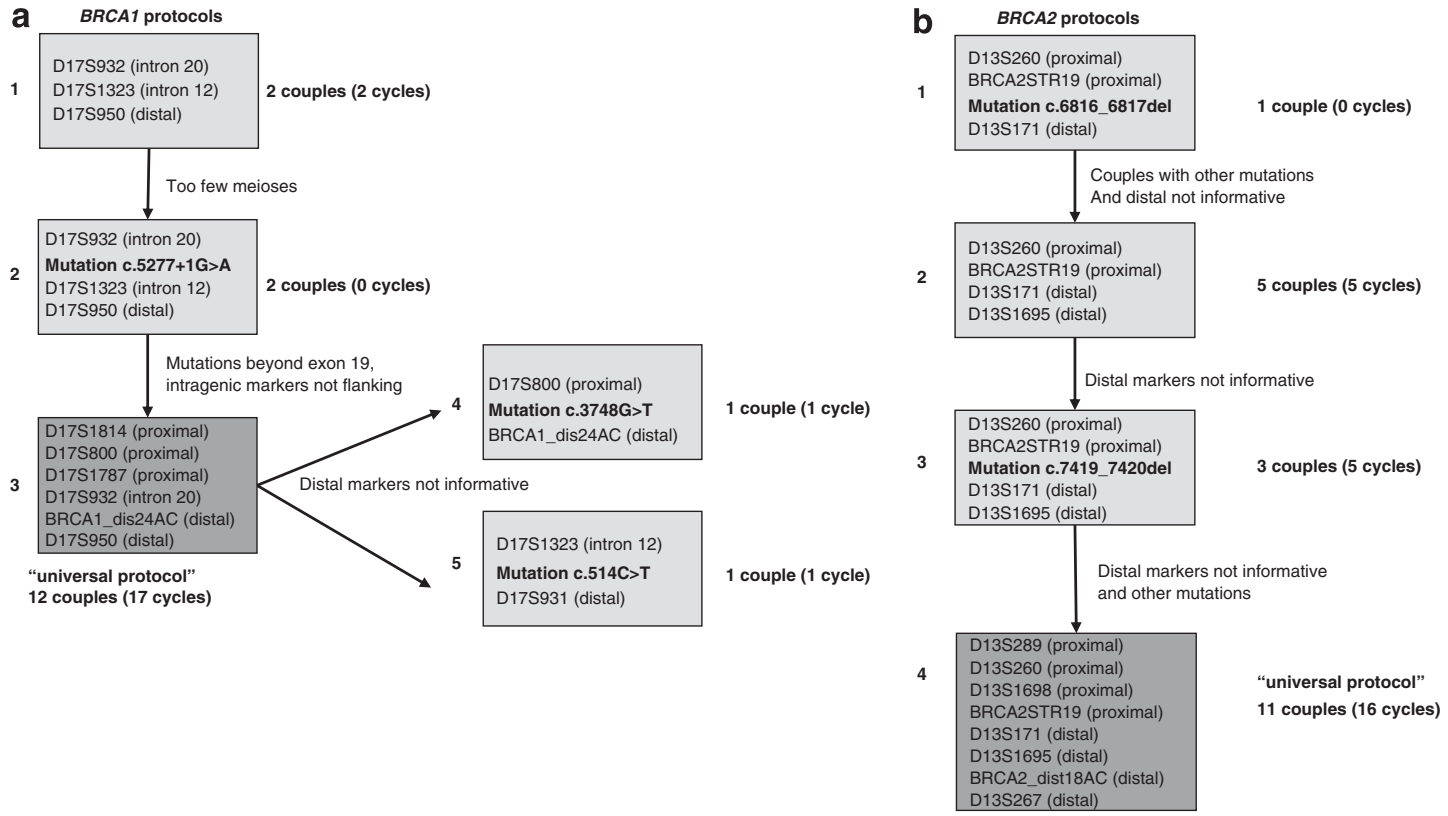

Figure 1 PGD protocols. Time-line overview of the developed single-cell PGD-PCR protocols for BRCA1 and BRCA2 mutation carriers. (a) Developed protocols for BRCA1, (b) developed protocols for BRCA2.

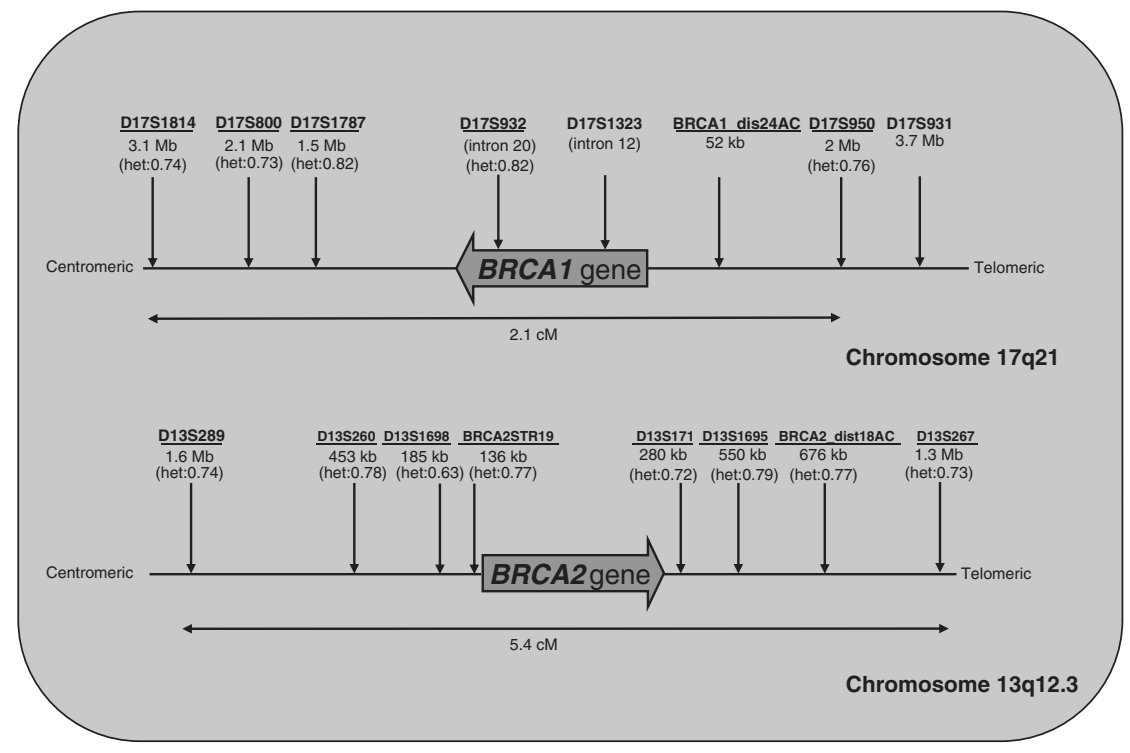

Figure 2 BRCA1 and BRCA2 loci and markers. Schematic overview of the BRCA1 and BRCA2 locus on chromosomes 17 and 13 , respectively. The large gene arrows indicate the direction of the loci with respect to chromosome orientation. Marker names and their genetic (cm) and physical distances (Mb) to the BRCA loci are indicated in the figure. Genetic distance is based on the Genethon genetic map. Heterozygosity of marker alleles (if known) are indicated between brackets. Underlined markers are the markers used in the described universal tests.

be determined with certainty. Therefore the mutation was included (protocol 2, Figure 1a). Thereafter many more couples applied with mutations beyond exon 19, leading to non-flanking markers. To avoid repetitive redesign of the protocol, it was adapted to the universal 6 marker protocol (protocol 3, universal, Figure 1a). For two couples thereafter the distal markers of the universal protocol were not informative and mutation-specific protocols were developed (protocols 4 and 5, Figure 1a).

For $B R C A 2$, a first protocol with a mutation and three markers, two proximal, one distal, was set up (protocol 1, Figure 1b). Thereafter couples with different mutations applied and in many couples the one distal marker was not informative. The protocol was changed to four markers (protocol 2, Figure 1b). As for some additional couples the two distal markers were not informative, the specific mutation was built in the existing protocol (protocol 3, Figure $1 b$ ). In the last phase, four additional markers were added to increase informativity (protocol 4, universal, Figure $1 \mathrm{~b}$ ).

The universal PGD protocols were developed approximately 2 years after continuously changing protocols and were thereafter applied to all new PGD couples. A detailed description of the validation of these protocols is depicted in Table 3. Also for couples who had a first PGD cycle performed with an old protocol, the next cycles were adapted to the novel universal protocols (see Table 1 for details). Since their introduction, 12 of the 14 BRCA1 couple haplotypes (86\%) were 
Table 2 BRCA1 and BRCA2 markers, primers and labels for universal PGD tests

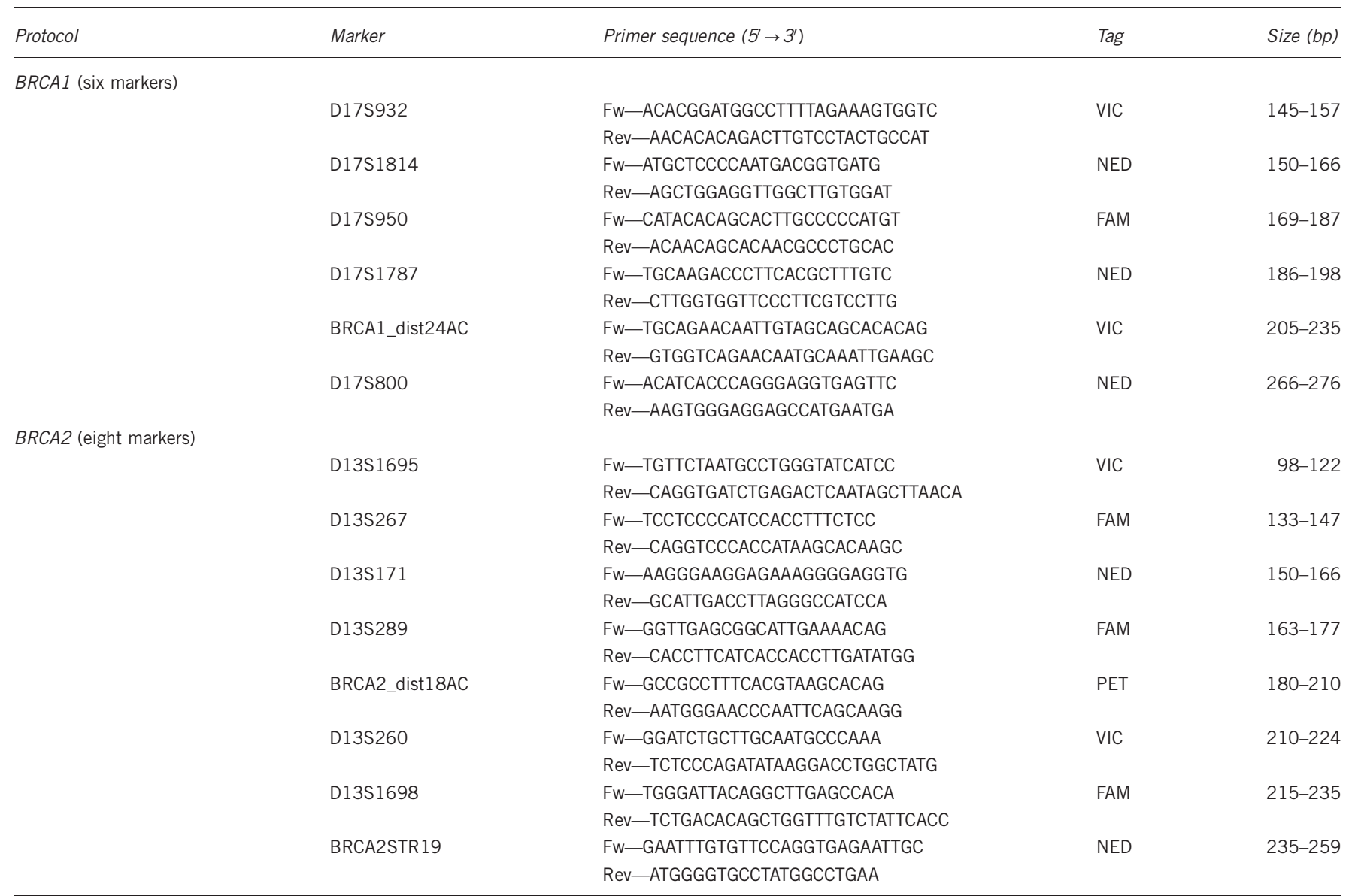

$\mathrm{FW}=$ forward, Rev $=$ reverse, $\mathrm{Tag}=$ fluorescent dyes tagged to $5^{\prime}$ end of forward primers

informative and all (100\%) BRCA2 family haplotypes were informative. These couples were ready to be scheduled for the PGD procedure within 1-2 months. For two PGD couples, the BRCA1 universal protocol was not applicable, because the markers at the distal side were not informative.

\section{Clinical cycles}

The results of the individual clinical cycles are summarized in Table 1. Thirty couples underwent a total of 47 cycles. Two of these 47 cycles concerned PGD analyses of embryos cryopreserved before biopsy in another IVF centre. Of the remaining 45 cycles, 8 cycles were cancelled before oocyte retrieval due to hyper-stimulation (2) or hypo-stimulation (6). In the 18 BRCA1 cycles, a total of 134 embryos were biopsied and a genetic diagnosis was obtained in 93\% (125/134); in the 21 BRCA2 cycles, a total of 127 embryos were biopsied and a genetic diagnosis was obtained in $93 \%$ (118/127). Of the embryos with a genetic diagnosis for BRCA1, 44.4\% carried the mutation, $35.7 \%$ were unaffected; for $B R C A 2,46.6 \%$ carried the mutation and $42.4 \%$ were unaffected. All remaining embryos were genetically abnormal or inconclusive.

In 34 of the 39 cycles (15 BRCA1/19 BRCA2, 87.2\%) an embryo transfer was possible and resulted in 10 pregnancies of which 8 were with a fetal heart beat ( $4 B R C A 1 / 4 B R C A 2)$, leading to a fetal heart beat per oocyte retrieval of $20.5 \%$ and a fetal heart beat per embryo transfer of $23.5 \%$. For the BRCA1 cycles, four pregnancies were obtained (see Table 1): Couple 3 delivered a healthy girl at $41^{+1}$ weeks with a birth weight of $3625 \mathrm{~g}$, Couple 6 delivered a healthy twin, a boy and a girl (two embryos transferred) with a birth weight of 3220 and $2660 \mathrm{~g}$ respectively. Couple 10 had an early miscarriage at 5 weeks. Couple 11 has an ongoing (single) pregnancy. For BRCA2 PGD couples, four pregnancies were obtained: Couple 3 delivered a healthy girl with a birth weight of $3120 \mathrm{~g}$ in August 2010 and is currently pregnant with a second child after a cryo-embryo transfer from the first cycle (at term 14 October 2012). Couple 4 delivered a healthy girl at $40^{+4}$ weeks with a birth weight of $2620 \mathrm{~g}$. The pregnancy of couple 15 is still ongoing. So far, none of the couples opted for prenatal diagnosis to confirm the determined unaffected genetic status in the PGD cycle.

\section{Quality assessment}

A total of 73 embryos were collected after PGD for re-analysis. In all, 13, 45 and 15 embryos were genotyped as unaffected, affected or aberrant during the PGD cycles. As the unaffected morphological good embryos are used for embryo transfer, the number of affected embryos succeeds the number of unaffected embryos in the re-analysis procedure. Of the 13 unaffected embryos, 11 showed conclusive genotype results after re-analysis. Two of 11 embryos gave one parental haplotype (monosomy), the non-risk marker haplotype 


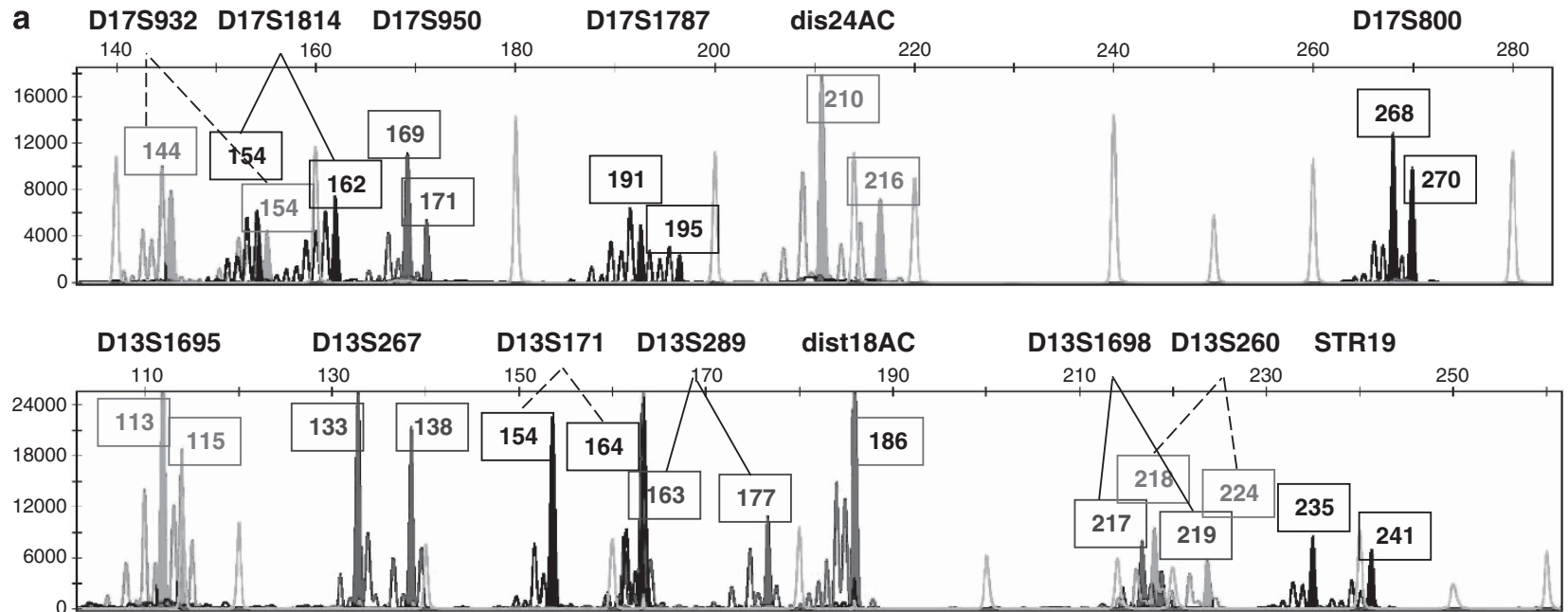

b

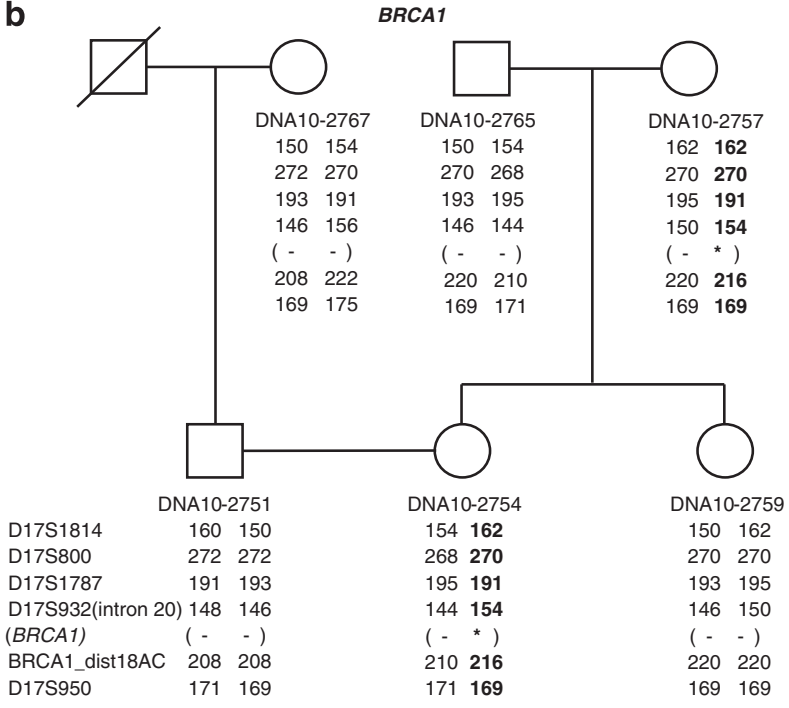

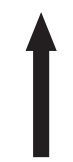
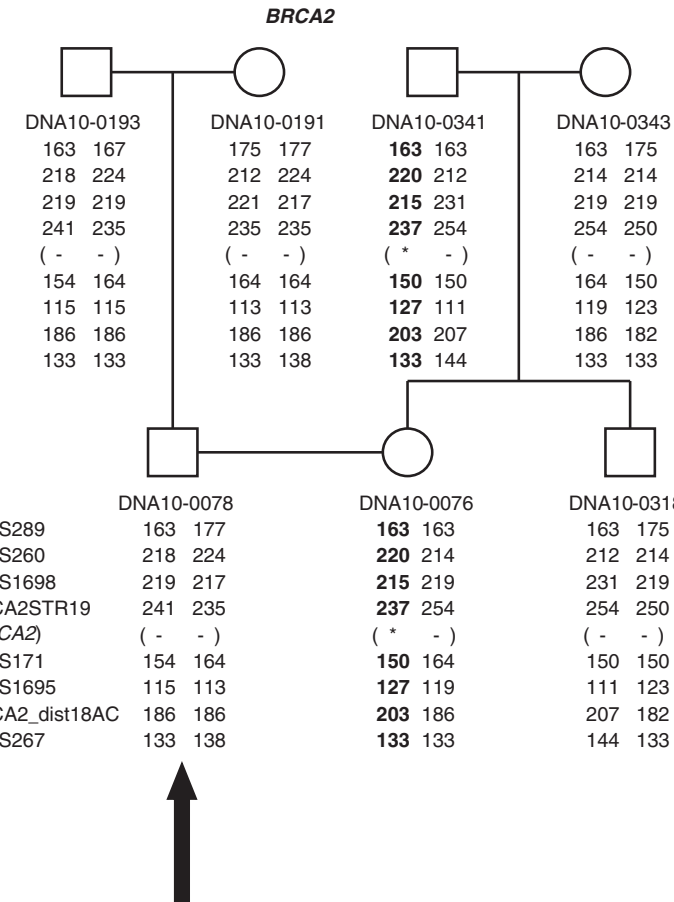

Figure 3 Haplotype analysis. (a) Genescan analysis example of a BRCA1 PGD index (patient) and a BRCA2 PGD index (partner). Absolute allele lengths are indicated in boxes, marker names above the diagrams. Colored peaks (see online colored version) indicate fluorescent labeling of VIC $=$ green, $N E D=b l a c k$, $\mathrm{FAM}=$ blue and PET=red. (b) Examples of a BRCA1 and BRCA2 family with haplotypes used in PGD cycles. Big black arrows represent patients corresponding to the genescan analysis in (a). Alleles in black represent the risk haplotypes co-segregating with the mutation. Asterisk indicates the position of the familial mutation.

of the affected parent. The two remaining embryos were not conclusive and could not be genotyped due to contamination after re-analysis. In $42 / 43$ (98\%) of the affected embryos, the PGD results were confirmed, one embryo gave no result; 5/15 aberrant embryos were confirmed $(33 \%)$, one monosomy gave no result, 7 monosomies were disomies and 2 trisomies were disomies in the re-analyses. Two recombinations were detected during the PGD cycles (Table 1), one around the BRCA1 locus and another around the BRCA2 locus. Both recombinations were confirmed in the re-analyses. The recombination around the BRCA1 locus crossed the two markers between which the mutation is located, and therefore the presence/absence of the mutation remained unknown in this embryo.

\section{DISCUSSION}

Since its introduction in the early nineties, PGD is considered a wellestablished clinical service in many countries for many human genetic diseases. $^{21}$ The range of indications has expanded from sex determination to prevention of chromosomal rearrangements to monogenic disorders. During the past few years, diseases of late age of onset and high (but not complete) penetrance with some therapeutic or preventive options such as HBOC have also been added to this list. PGD for this indication has therefore been a vigorous topic of debate in several countries ${ }^{22}$ in the past couple of years. Since 2008, several BRCA1/2 carrier couples applied for PGD. We started with a mutation-specific protocol with few markers and 
Table 3 Validation of universal protocols

\begin{tabular}{llccccc}
\hline & & No. of & & ADO & PCR & Positive \\
single & & & (\%) & efficiency & blanks \\
cells & No. of & per & (\%) per & per \\
Gene & Marker & tested & heterozygotes & locus & locus & locus \\
\hline \multirow{2}{*}{ BRCA1 } & D17S1814 & 57 & 54 & 1.8 & 96.5 & $0 / 13$ \\
& D17S800 & 57 & 55 & 1.8 & 96.5 & $0 / 13$ \\
& D17S1787 & 57 & 55 & 1.8 & 96.5 & $0 / 13$ \\
& D17S932 & 57 & 55 & 1.8 & 96.5 & $0 / 13$ \\
& BRCA1_dis24AC & 57 & 55 & 0.0 & 96.5 & $1 / 13$ \\
D17S950 & 57 & 54 & 0.0 & 96.5 & $0 / 13$ \\
BRCA2 & D13S289 & 106 & 104 & 1.9 & 98.1 & $0 / 24$ \\
D13S260 & 106 & 69 & 5.8 & 98.1 & $0 / 24$ \\
D13S1698 & 106 & 104 & 5.8 & 98.1 & $0 / 24$ \\
BRCA2STR19 & 106 & 103 & 1.9 & 97.2 & $0 / 24$ \\
D13S171 & 106 & 35 & 2.9 & 98.1 & $0 / 24$ \\
D13S1695 & 106 & 34 & 0.0 & 97.2 & $1 / 24$ \\
BRCA2_dist18AC & 106 & 70 & 0.0 & 97.2 & $0 / 24$ \\
D13S267 & 106 & 69 & 0.0 & 98.1 & $0 / 24$ \\
\hline
\end{tabular}

protocols with some informative markers for first couples. These protocols were not applicable to all couples and thus were adapted by either replacing the mutation-specific primers or adding/replacing markers. Also in the literature the same laborious strategy is visible; Spits et al. ${ }^{13}$ have described a protocol for BRCA1 using two markers, flanking the BRCA1 locus. These markers are both intragenic, making the protocol suitable only if both markers are informative and the mutation itself is located between the two intragenic markers. Jasper et al. ${ }^{23}$ have included one specific mutation in BRCA1 combined with two proximal markers but used two rounds of PCR. Sagi et al. ${ }^{12}$ have described one BRCA1 and two BRCA2 founder-mutation-specific protocols, including three or four markers flanking the $B R C A$ loci, applied to 10 PGD couples from Israel, and finally Ramon et al. ${ }^{24}$ have described one mutation-specific BRCA1 protocol with two markers. These protocols are not universal as they are mutation specific and/or include only a very small number of markers limiting informativity and using intragenic markers creates the limitation that the mutation must be located between the markers. Additionally, it takes a lot of time to design and optimize multiplex single-cell PCRs to maximize PCR efficiency and minimize ADO percentages for each specific mutation. To circumvent this costly and laborious procedure, other strategies have already been illustrated. Pre-implantation genetic 'haplotyping' has been described as a more universal strategy for several inherited monogenic disorders. ${ }^{25}$ However, this strategy uses an extra step of multiple displacement amplification (MDA) of the single cell. This additional step is needed to allow a combination of several multiplexed PCRs in the following step, which would otherwise not be feasible on a single blastomere. This principle is excellent in the sense that it is applicable to all familiar monogenic diseases and using several multiplexed PCRs will optimize informativity of markers and detection of recombinations. One major drawback of MDA is, however, the high percentage of $\mathrm{ADO}$, which needs to be compensated with a higher number of markers tested. A second drawback can be the extra time needed (16h) to perform the MDA, which may put pressure on the timing of embryonic transfer if transfer is performed on day 4 post fertilization (which is the case in our centre). Therefore, for centres that transfer at day 5 , the time needed to do MDA is not an issue. We developed an 'off-the-shelf' and fast test for BRCA1 and BRCA2 mutation carriers applicable to nearly all couples; we designed one multiplexed PCR test, including as much as possible flanking informative markers, working on a single biopsied blastomere.

For the BRCA1 locus, we were able to multiplex six highly polymorphic markers in one PCR. The maximal distance of the two outer markers is $2.1 \mathrm{~cm}$. Three markers are located in the proximal region, two in the distal region and one is located intragenic (intron 20). For the BRCA2 locus, we were able to multiplex eight highly polymorphic markers in one PCR, with a maximal distance between the outer markers of $4.65 \mathrm{~cm}$. All eight markers are flanking the BRCA2 locus, four proximally and four distally. As mentioned earlier, we started out with approximately 20-30 markers per locus in the early stages of development. Taking several criteria (heterozygosity, ADO percentage, competition, etc) as well as single-cell PCR limitations into account, the final number as well as the specific location of markers chosen in the universal protocols are not unique; other numbers or combinations of markers would be possible as long as they meet the same validation criteria and are informative in the majority of new couples.

Eighty-six percent of the BRCA1 carriers and all BRCA2 mutation carriers could so far be analyzed with the universal tests. If the pedigrees of the 'old protocol' cycles are reassessed and the universal protocols would have been used, 13/15 BRCA1 couples (87\%) and 14/15 (93\%) were informative. Only two BRCA1 PGD couples were not informative at the distal side and one BRCA2 couple would not have been informative at the distal side. Preparation time is limited to 1-2 months, let alone the cost reduction saved by using the 'off-the-shelf' tests.

The robustness of the multiplex BRCA PCR tests are in accordance with the latest edition of the ESHRE PGD consortium guidelines, ${ }^{16}$ with a PCR efficiency $>90 \%$ and ADO rates $<10 \%$.

\section{CONCLUSIONS}

In summary, we present an universal 'off-the-shelf' PGD tests for $B R C A 1$ and BRCA2 mutation carriers that are robust, easy and quick to implement for a wide range of families choosing PGD to avoid transmission of a $B R C A 1 / 2$ mutation to future offspring.

\section{CONFLICT OF INTEREST}

The authors declare no conflict of interest.

\section{ACKNOWLEDGEMENTS}

We thank other members of the PGD working group who contributed to the study: Marieke van Deursen, Yvonne Arens, Chantal Bastiaens, Celine Eggen, Nienke Muntjewerff, Guusje de Krom, Aisha de Graaff, Ewka Nelissen, Inge Schreurs, Marij Janssen, Mirjam Wolffs, Marion Meijs, Josien Derhaag, John Dumoulin, Sabine Spierts, Wim Loneus, Yens Jackers, Laurence Meers, Anneke de Vreeden-Elbertse, Yvonne Koot, Madelon Meijer-Hoogeveen, Irene Homminga, Elsbeth Dul and Jolande Land.

1 Devilee P, Tollenaar RA, Cornelisse CJ: [From gene to disease; from BRCA1 or BRCA2 to breast cancer]. Ned Tijdschr Geneeskd 2000; 144: 2549-2551.

2 Tutt A, Ashworth A: The relationship between the roles of BRCA genes in DNA repair and cancer predisposition. Trends Mol Med 2002; 8: 571-576.

3 Ford D, Easton DF, Bishop DT, Narod SA, Goldgar DE: Risks of cancer in BRCA1mutation carriers. Breast Cancer Linkage Consortium. Lancet 1994; 343: 692-695.

4 Wooster R, Bignell G, Lancaster J et al: Identification of the breast cancer susceptibility gene BRCA2. Nature 1995; 378: 789-792.

5 Abeliovich D, Kaduri L, Lerer I et al: The founder mutations 185 delAG and 5382 insC in BRCA1 and 6174 delT in BRCA2 appear in $60 \%$ of ovarian cancer and $30 \%$ of earlyonset breast cancer patients among Ashkenazi women. Am J Human Genet 1997; 60: 505-514. 
6 Borg A, Dorum A, Heimdal K, Maehle L, Hovig E, Moller P: BRCA1 1675delA and 1135 ins A account for one third of Norwegian familial breast-ovarian cancer and are associated with later disease onset than less frequent mutations. Dis Markers 1999, 15: 79-84.

7 Gorski B, Jakubowska A, Huzarski T et al: A high proportion of founder BRCA1 mutations in Polish breast cancer families. IntJ Cancer 2004; 110: 683-686.

8 Khoo US, Chan KY, Cheung AN et al: Recurrent BRCA1 and BRCA2 germline mutations in ovarian cancer: a founder mutation of BRCA1 identified in the Chinese population. HumanMmutat 2002; 19: 307-308.

9 Verhoog LC, van den Ouweland AM, Berns $E$ et al: Large regional differences in the frequency of distinct BRCA1/BRCA2 mutations in 517 Dutch breast and/or ovarian cancer families. Eur J Cancer 2001; 37: 2082-2090.

10 de Die-Smulders CE, Land JA, Dreesen JC, Coonen E, Evers JL, Geraedts JP: [Results from 10 years of preimplantation-genetic diagnostics in The Netherlands]. Ned Tijdschr Geneeskd 2004; 148: 2491-2496.

11 Menon U, Harper J, Sharma A et al: Views of BRCA gene mutation carriers on preimplantation genetic diagnosis as a reproductive option for hereditary breast and ovarian cancer. Hum Reprod 2007; 22: 1573-1577.

12 Sagi M, Weinberg N, Eilat A et al: Preimplantation genetic diagnosis for BRCA1/2-a novel clinical experience. Prenat Diagn 2009; 29: 508-513.

13 Spits $\mathrm{C}$, De Rycke M, Van Ranst $\mathrm{N}$ et al: Preimplantation genetic diagnosis for cancer predisposition syndromes. Prenat Diagn 2007; 27: 447-456.

14 Kotsopoulos J, Librach CL, Lubinski J et al: Infertility, treatment of infertility, and the risk of breast cancer among women with BRCA1 and BRCA2 mutations: a case-control study. Cancer Causes Control 2008; 19: 1111-1119.

15 Moutou C, Gardes N, Nicod JC, Viville S: Strategies and outcomes of PGD of familial adenomatous polyposis. Mol Hum Reprod 2007; 13: 95-101.
16 Harton GL, De Rycke M, Fiorentino F et al: ESHRE PGD consortium best practice guidelines for amplification-based PGD. Hum Reprod 2011; 26: 33-40.

17 Dumoulin JC, Land JA, Van Montfoort AP et al: Effect of in vitro culture of human embryos on birthweight of newborns. Hum Reprod 2010; 25: 605-612.

18 Van Steirteghem AC, Nagy Z, Joris $\mathrm{H}$ et al: High fertilization and implantation rates after intracytoplasmic sperm injection. Hum Reprod 1993; 8: 1061-1066.

19 Dumoulin JC, Coonen E, Bras M et al: Comparison of in-vitro development of embryos originating from either conventional in-vitro fertilization or intracytoplasmic sperm injection. Hum Reprod 2000; 15: 402-409.

20 Thornhill AR, deDie-Smulders CE, Geraedts JP et al: ESHRE PGD Consortium 'Best practice guidelines for clinical preimplantation genetic diagnosis (PGD) and preimplantation genetic screening (PGS)'. Hum Reprod 2005; 20: 35-48.

21 Handyside $\mathrm{AH}$ : Preimplantation genetic diagnosis after 20 years. Reprod Biomed Online 2010; 21: 280-282.

22 Robertson JA: Extending preimplantation genetic diagnosis: the ethical debate Ethical issues in new uses of preimplantation genetic diagnosis. Hum Reprod 2003; 18: 465-471.

23 Jasper MJ, Liebelt J, Hussey ND: Preimplantation genetic diagnosis for BRCA1 exon 13 duplication mutation using linked polymorphic markers resulting in a live birth. Prenat Diagn 2008; 28: 292-298.

24 Ramon YCT, Polo A, Martinez 0 et al: Preimplantation genetic diagnosis for inherited breast cancer: first clinical application and live birth in Spain. Familial Cancer 2012 11: $175-179$.

25 Renwick PJ, Trussler J, Ostad-Saffari E et al: Proof of principle and first cases using preimplantation genetic haplotyping - a paradigm shift for embryo diagnosis. Reprod Biomed Online 2006; 13: 110-119. 